\title{
Sindrome de Aspiração do Mecônio: Análise de Resultados Obstétricos e Perinatais
}

\author{
Meconium Aspiration Syndrome: Obstetric and Perinatal Outcome Analysis
}

José Mauro Madi, Edson Nunes de Morais

Erinéia Locatelli, Sônia Regina Cabaral Madi, Renato Luis Rombaldi

\begin{abstract}
RESUM0
Objetivos: analisar os resultados obstétricos e perinatais de 26 casos de sindrome de aspiração de mecônio (SAM).

Métodos: realizou-se revisão dos prontuários de 26 recém-nascidos (RN) com diagnóstico de SAM. Os casos foram estudados em função da média de permanência do RN na UTIN e das principais complicações maternas e neonatais, correlacionando-as entre si.

Resultados: dezoito conceptos nasceram no HG-FUCS e 8 fora; no periodo citado, ocorreram 3.976 nascimentos no HG-FUCS, incidência de SAM de 0,45\%. Dos 18 casos estudados, 9 nasceram pela via vaginal; o peso ao nascimento foi $\geq 2.500 \mathrm{~g}$ em dezesseis casos. Mecônio leve ocorreu em 50\%, semelhante ao espesso. O Apgar no $1^{\circ}$ minuto foi $\geq 7 \mathrm{em} 3$ casos (16,7\%), entre 4 e 6, em 7 casos (38,9\%), e entre 0 e 3, em 8 casos (44,4\%). No $5^{\circ}$ minuto, sete RN permaneceram deprimidos. A principal complicação neonatal foi anoxia (36\% dos casos). A taxa de óbito neonatal foi de 7,7\%. A internação média na UTIN foi de 19,9 dias.

Conclusão: a SAM constitui grave entidade clinica neonatal, relacionando-se com altas taxas de mortalidade neonatal, mecônio espesso em pelo menos a metade dos casos e nascimento de fetos deprimidos na sua maioria.
\end{abstract}

PALAVRAS-CHAVE: Aspiração do mecônio. Mecônio. Mortalidade neonatal. Asfixia intra-uterina.

\section{Introdução}

A incidência da eliminação de mecônio durante o trabalho de parto tem sido descrita na literatura variando de 0,5 a $30 \%$, a depender do local onde foram realizados os estudos, do tipo de população estudada e dos critérios diagnósticos do momento de eliminação do mecônio ${ }^{1-3}$. Se por um lado o fenômeno é freqüente, o risco de complicações associadas é relativamente baixo, ainda que a sua ocorrência esteja relacionada a elevadas taxas de morbidade e mortalidade neonatal ${ }^{4}$. Basicamente, é uma forma de pneumonia de aspi-

Serviço de Obstetrícia e Unidade de Tratamento Intensivo Neonatal (UTIN) do Hospital Geral de Caxias do Sul Fundação Universidade de Caxias do Sul (HG-FUCS). Correspondência:

José Mauro Madi

CCBS/UCS - Cidade Universitária.

95070-560 - Caxias do Sul - RS

Telefone e fax: (54) 229-4444

e-mail: madi@visao.com.br ração passivel de ocorrer principalmente em gestações de termo. O índice de mortalidade perinatal pode variar de 10 a $20 \%$, embora alguns autores refiram taxas de até $40 \%$, mesmo colocando-se em prática o manejo agressivo do recém-nascidos (RN) imediatamente após o parto ${ }^{1-4}$. O conceito de que a aspiração ocorre com a primeira incursão respiratória e o de que a aspiração do orofaringe deva ser realizada logo após o desprendimento da cabeça fetal no períneo materno são questionados, pois cerca de $35 \%$ dos conceptos apresentam mecônio abaixo das cordas vocais ${ }^{5}$.

Enfim, a justificativa principal para a investigação realizada está na gravidade da síndrome da aspiração do mecônio (SAM), quase sempre associada a fetos de termo e com peso adequado para a idade gestacional, e na íntima associação da doença a altos índices de operação cesariana, depressão neonatal grave, resultados perinatais adversos e incremento nas taxas de internação em ambiente de intensivismo neonatal. 
O objetivo do presente trabalho foi o de estudar, retrospectivamente, casos de sindrome de aspiração de mecônio ocorridos em população de um hospital-escola, sob os aspectos obstétricos e neonatais.

\section{Pacientes e Métodos}

Realizou-se estudo retrospectivo em $26 \mathrm{RN}$ internados na UTIN do HG-FUCS com diagnóstico de SAM. Para fins de análise e discussão da doença em questão, consideraram-se apenas os 18 prontuários de conceptos nascidos no Serviço de Obstetrícia do HG-FUCS. Os 26 casos foram incluídos no estudo da média de permanência do RN na UTIN e das principais complicações neonatais. A maioria das gestantes era primigesta $(55,6 \%)$, compareceu a consultas de pré-natal em número superior a $5(61,1 \%)$ e não era tabagista $(77,8 \%)$. A incidência de rotura prematura de membranas amnióticas foi de $16,7 \%$ e o uso de ocitocina durante o trabalho de parto foi de $66,7 \%$. O parto ocorreu por via transpélvica em metade das vezes. Foram analisadas: intensidade de mecônio por ocasião do parto (leve e espesso), o peso do RN, a adequação do peso fetal, o índice de Apgar de $1^{\circ} \mathrm{e}$ $5^{\circ}$ minuto, as condições de alta do neonato, as principais complicações neonatais e o tempo de internação hospitalar do neonato.

O período do estudo foi de março de 1998 a julho de 2001. Nesse periodo, ocorreram 3.976 nascimentos. Considerou-se como sofrimento fetal a presença de alteração da freqüência cardíaca fetal (FCF) no registro cardiotocográfico, segundo os critérios do "National Institutes of Child Health" $(1997)^{4}$; como RN com depressão leve aquele que apresentasse Apgar ente 4 e 6 , e grave abaixo de 4 no $1^{\circ} \mathrm{e} /$ ou $5^{\circ}$ minuto. Para o diagnóstico de SAM foram consideradas as presenças de mecônio nas cordas vocais do $\mathrm{RN}$, além de sinais clínicos e radiológicos observados na UTIN. A análise dos resultados foi realizada por meio de estatística descritiva.

Esse estudo não apresentou riscos aos indivíduos envolvidos, posto tratar-se de pesquisa retrospectiva; mesmo assim, obteve aprovação da Comissão de Ética da Universidade de Caxias do Sul.

\section{Resultados}

Os resultados do estudo serão apresentados sob a forma de tabelas. Nas Tabelas 1 e 2 encontram-se os dados gerais dos $18 \mathrm{RN}$ que desenvolveram SAM. Observa-se que o número de casos foi semelhante entre os conceptos nascidos de partos vaginais e/ou cesáreos. Por outro lado, a taxa de $50 \%$ de cesárea nesses casos é significativamente superior àquela da população geral do HG-FUCS (20,8\% no período em estudo). Em relação às condições de alta do RN, é notável o número de neomortos $(11,1 \%)$. A grande maioria dos neonatos era de termo e com peso superior a 2.500 g $(88,8 \%)$, bem como era adequada para a idade gestacional $(83,3 \%)$.

Tabela 1 - Dados gerais de 18 casos de síndrome de aspiração do mecônio no HG-FUCS.

\begin{tabular}{lrr}
\hline Dados gerais & $\mathbf{n}$ & $\%$ \\
\hline Idade gestacional & & \\
RN* de termo & 17 & 94,4 \\
RN pré-termo & 1 & 5,6 \\
Peso RN & & \\
$\geq 2.500 \mathrm{~g}$ & 16 & 88,8 \\
$1500-2.500 \mathrm{~g}$ & 1 & 5,6 \\
$<1500 \mathrm{~g}$ & 1 & 5,6 \\
Adequação do peso fetal & & \\
AlG** & 15 & 53,3 \\
PIG*** & 1 & 11,1 \\
GIG*** & 2 & 77,8 \\
Condições de alta & & 11,1 \\
Boas condições & 14 & \\
Óbito & 2 &
\end{tabular}

${ }^{*} \mathrm{RN}=$ Recém-nascido

** AIG = Adequado para a idade gestacional

${ }^{* * *} P I G=$ Pequeno para a idade gestacional

**** $\mathrm{GIG}=$ Grande para a idade gestacional

Tabela 2 - Indicadores de sofrimento fetal de 18 casos de síndrome de aspiração do mecônio no HG-FUCS.

\begin{tabular}{|c|c|c|}
\hline & $\mathrm{n}$ & $\%$ \\
\hline \multicolumn{3}{|c|}{ Tipo de mecônio } \\
\hline Leve & 9 & 50,0 \\
\hline Espesso & 9 & 50, \\
\hline \multicolumn{3}{|c|}{ Apgar de $1^{\circ}$ minuto } \\
\hline 7 a 10 & 3 & 16,7 \\
\hline 4 a 6 & 7 & 38, \\
\hline 0 a 3 & 8 & 44, \\
\hline \multicolumn{3}{|c|}{ Apgar de $5^{\circ}$ minuto } \\
\hline 7 a 10 & 11 & 61, \\
\hline 4 a 6 & 6 & 33,3 \\
\hline 0 a 3 & 1 & 5,6 \\
\hline \multicolumn{3}{|c|}{ Sofrimento fetal } \\
\hline Presente & 14 & 77 \\
\hline Ausente & 4 & 22,2 \\
\hline
\end{tabular}

$\mathrm{O}$ indice de Apgar no $1^{\circ}$ minuto mostrou que apenas $16,7 \%$ dos RN eram vigorosos. Já no $5^{\circ}$ minuto, ainda permaneciam deprimidos 38,9\% deles. O diagnóstico de sofrimento fetal (cardiotocografia alterada) esteve presente em $77,8 \%$ dos casos. 
A Tabela 3 relaciona a ocorrência de sofrimento fetal com a intensidade de mecônio e o índice de Apgar de $1^{\circ}$ e $5^{\circ}$ minuto. Merecem ênfase as altas incidências de mecônio espesso $(57,1 \%)$ e Apgar $<7(78,6 \%)$ no $1^{\circ}$ minuto, associadas à dificil recuperação do RN no $5^{\circ}$ minuto $(42,8 \%)$. Por outro lado, é de se notar que, na ausência de sofrimento fetal (cardiotocografia normal), o mecônio era leve na maioria dos casos $(75 \%)$, ainda que a maioria dos $\mathrm{RN}$ apresentasse Apgar inferior a 7 no $1^{\circ}$ minuto $(75 \%)$, recuperando-se em grande parte no $5^{\circ}$ minuto $(75 \%)$.

Tabela 3 - Distribuição de 18 casos com síndrome de aspiração do mecônio em função da ocorrência de sofrimento fetal intraparto, intensidade de mecônio e Apgar de $1^{\circ}$ e $5^{\circ}$ minuto.

\begin{tabular}{lccccccc} 
& & \multicolumn{2}{c}{ Mecônico } & \multicolumn{2}{c}{ Apgar 10 minuto } & \multicolumn{2}{c}{ Apgar 5 minuto } \\
& & Espesso & Leve & $<7$ & $\leq 7$ & $<7$ & $\leq 7$ \\
\hline C/ SF $^{*}$ & $n$ & 8 & 6 & 11 & 3 & 6 & 8 \\
& $\%$ & 57,1 & 42,9 & 78,6 & 21,4 & 42,8 & 57,2 \\
$\mathrm{~S} \mathrm{SF}^{* *}$ & $n$ & 1 & 3 & 3 & 1 & 1 & 3 \\
& $\%$ & 25 & 75 & 75 & 25 & 25 & 75
\end{tabular}

* com sofrimento fetal

** sem sofrimento fetal

Na Tabela 4 encontram-se arroladas as principais complicações neonatais dos 26 RN com SAM. Observa-se que a maioria não apresentou complicações $(53,8 \%)$. Dentre os que apresentaram complicações, a anoxia neonatal leve ou grave ocorreu em $47,2 \%$, ao passo que as convulsões foram observadas em $15,4 \%$ dos casos.

Tabela 4 - Principais complicações neonatais de 26 RN com síndrome de aspiração do mecônio.

\begin{tabular}{lc}
\hline Complicações & $\%$ \\
\hline Nenhuma & 53,8 \\
Anoxia leve & 27,0 \\
Anoxia grave & 19,2 \\
Sepse & 15,4 \\
Convulsões & 15,4 \\
Outras & 19,0 \\
\hline
\end{tabular}

Média de permanência na UTIN: 19,9 dias

Dos RN referidos para o HG-FUCS (8 casos), ocorreu 1 morte neonatal, ao passo que 6 tiveram alta hospitalar em boas condições. Todos eram de termo, com peso superior a $2.500 \mathrm{~g}$ e adequados para a idade gestacional. A média de internação desses 8 RN foi de 12,4 dias vs 19,9 dias do total de 26 RN internados na UTIN.

\section{Discussão}

A presença de mecônio no líquido amniótico é considerada para alguns como evento decorrente de um quadro hipóxico preexistente, eventual ou não, e, em conseqüência, pode ser interpretado como um marcador de comprometimento fetal prévio ${ }^{1}$.
Houlihan e Knuppel ${ }^{2}$, assim como Morais ${ }^{3}$, referem que o mecônio no líquido amniótico pode estar presente em 9 a $20 \%$ dos partos, podendo chegar a $30 \%$, dependendo do serviço onde o estudo foi realizado. Em hospitais de referência terciária, aos quais os casos de doença obstétrica são mais freqüentemente referidos, costuma-se identificar incidência aumentada do evento.

$\mathrm{Na}$ casuística do HG-FUCS, quando houve detecção de sofrimento fetal intraparto, a incidência de mecônio leve e espesso foi de $42,9 \%$ e de $57,1 \%$, respectivamente. Ao contrário, quando não houve sofrimento fetal associado, o percentual foi de 75 e $25 \%$. O índice de Apgar de $1^{\circ}$ minuto $<7$ $(78,6 \%)$ esteve também correlacionado com a presença de mecônio espesso. Yong e $\mathrm{Ho}^{5}$ revisaram a incidência de mecônio espesso por ocasião do parto e SAM. Para tal, estudaram, retrospectivamente, 1.893 recém-nascidos, cujos partos estiveram relacionados com a presença de mecônio (15\% dos nascidos vivos) e 174 (1,4\% dos nascidos vivos) que apresentaram SAM. A incidência de mecônio leve, moderado e espesso foi de 0,3, 5,8 e $61 \%$, respectivamente. A taxa de mortalidade foi de $2,0 \%$ e a SAM associou-se ao mecônio espesso. Nathan et al. ${ }^{6}$ referiram ser o mecônio no líquido amniótico evento obstétrico de baixa incidência, mas correlacionado com substancial risco de resultados perinatais adversos, quando comparados com os partos que cursaram com líquido claro.

Nesse estudo, ao se comparar líquido amniótico claro e meconial, os autores observaram que a mortalidade perinatal (quase sempre resultante de SAM) evolveu de 0,3\%o para 1,5\%o, a acidemia grave fetal $(\mathrm{pH}$ de artéria umbilical $<7,00$ ) aumentou de $3,0 \%$ o para $7,0 \%$, ao passo que a incidência de cesárea passou de $7 \%$ para $14 \%$, respectivamente. 
Na presente análise, o mecônio espesso foi detectado em 9 partos, sendo que em 8 esteve associado ao sofrimento fetal.

O parto cesáreo foi realizado na metade dos casos $(50 \%)$, ou seja, 2,5 vezes maior que a incidência de cesárea na população obstétrica geral do HG-FUCS, no mesmo período de tempo $(20,8 \%)$. Merece destaque o estudo de Benny et al. (1987) que cita a ocorrência de SAM em 66 de um total de 120 conceptos que, por ocasião do nascimento, cursaram com líquido âmnico meconial. Dos 66 afetados, 4 morreram e 17 necessitaram de suporte ventilatório. Nessa pesquisa, quando da ocorrência de quadro grave de SAM, observaram associação significativa dessa doença com gestantes tabagistas. A casuística apresentada pelo HG-FUCS mostra que o tabagismo esteve presente em somente $22 \%$ dos casos.

Urbaniak et al. ${ }^{8}$ objetivaram identificar fatores de risco para a SAM nos períodos antenatal e intraparto que pudessem ser prevenidos; para tal, analisaram 210 neonatos que apresentaram mecônio no líquido amniótico e que desenvolveram distúrbios respiratórios no pós-parto imediato. A incidência de SAM foi de $6,6 \%$ em crianças expostas ao mecônio, e de 1,3\% na população geral de nascimentos. Os autores encontraram associação significante entre SAM e primiparidade, bem como entre a intensidade do mecônio e baixo $\mathrm{pH}$ da artéria umbilical. O mecônio espesso e a cardiotocografia anormal foram mais comumente observadas em SAM grave. Todas os óbitos e morbidades observados a longo prazo ocorreram em crianças que apresentaram FCF anormal, ou que não foram monitorizadas. Não foram identificados fatores de risco antenatais e intraparto.

Adhikari et al. ${ }^{9}$ relatam que a cardiotocografia intraparto para o diagnóstico de comprometimento fetal foi o fator que mais contribuiu para a prevenção e redução da SAM. A presença de alterações radiológicas graves nos RN que foram submetidos à aspiração traqueal e que apresentaram $\mathrm{pH}$ da artéria umbilical mais baixo mostrou que a aspiração ocorre, em alguns casos, antes do parto. Os autores sugerem que, em virtude do número de crianças nascidas pós-termo (54\%) em sua casuística, deve-se evitar a pós-maturidade como fator preventivo para SAM. Em nosso material não se observou nenhum caso de pós-datismo; ao contrário, $17 \mathrm{RN}$ eram de termo $(94,4 \%)$ e somente 1 era pré-termo $(5,6 \%)$.

Ao considerar os 26 RN que tiveram o diagnóstico de SAM no HG-FUCS, ver-se-á que a morbidade neonatal esteve correlacionada com anoxia leve $(27 \%)$, anoxia grave $(19,2 \%)$, sepse $(15,4 \%)$, crises convulsivas $(15,4 \%)$ e seqüelas neurológicas $(11,1 \%)$.
Usta et al. ${ }^{10}$, ao estudarem $937 \mathrm{RN}$, dos quais 39 desenvolveram SAM, objetivando identificar indicadores em potencial para a doença aspirativa em gravidezes complicadas por mecônio moderado ou espesso, observaram a existência de 6 variáveis independentes e com efeito estatisticamente significante sobre a SAM: indução de partos com traçados cardiotocográficos não reativos, necessidade de entubação endotraqueal, aspiração do líquido acumulado abaixo das cordas vocais, Apgar $£ 4$ no $1^{\circ}$ minuto, parto cesáreo e parto cesáreo prévio. A presença de pelo menos 1 dos 5 fatores de risco mostrou sensibilidade de $92 \%$, especificidade de $56 \%$, valor preditivo positivo de $8 \%$ e valor preditivo negativo de 99\% para a SAM. Em nossa casuística, observou-se índice de Apgar $<7$ no $1^{\circ}$ minuto em 15 casos (83,3\%); no $5^{\circ}$ minuto, $7 \mathrm{RN}$ permaneciam com Apgar $<7$ (38,9\%). Se se considerar a depressão leve e grave, observar-se-á Apgar de $1^{\circ}$ minuto entre 0 e 3 em 8 casos $(44,4 \%)$ e entre 4 e 6 , em 7 casos $(38,9 \%)$. No $5^{\circ}$ minuto, entre 0 e 3 , foi observado 1 caso $(5,6 \%)$, e entre 4 e 6,6 casos $(33,3 \%)$.

A casuística do HG-FUCS mostrou dois aspectos importantes: (i) 10 pacientes eram primigestas $(55,6 \%)$; (ii) os pesos dos conceptos variaram de $<1.500$ a 2.500 g em 2 casos $(11,2 \%)$ e estiveram superior a $2.500 \mathrm{~g}$ em 16 casos $(88,8 \%)$. A despeito de $15 \mathrm{RN}$ apresentarem peso adequado para a idade gestacional (83,3\%), o Apgar dos RN acometidos esteve freqüentemente baixo, ou seja, a SAM, de modo geral, parece estar relacionada com nascituros de termo e com peso apropriado, constituindo evento desagradável tanto para o obstetra como para a parturiente. Esses dados são similares a de outros autores.

As manobras obstétricas e neonatais parecem se associar com os resultados neonatais em casos de SAM. Chishty et al. ${ }^{11}$, em estudo comparativo entre 2 grupos de RN (nascidos no serviço dos autores e fora dele, mas ambos os grupos submetidos a entubação endotraqueal e ressuscitação), identificaram, entre outras variáveis, significativas diferenças no tempo de permanência no hospital ( 5 dias vs 2,87 dias). O tempo médio de permanência dos fetos nascidos no HG-FUCS foi de 19,9 dias, ao passo que o dos nascidos fora dele foi de 12,4 . Não se analisou a relação descrita pelos autores anteriormente citados (entubação endotraqueal e ressuscitação), ao mesmo tempo em que a provável diferença nas amostras estudadas pode explicar as diferenças de internação média encontradas.

Um estudo retrospectivo levado a efeito por 11 instituições na África do Sul revelou a alta incidência da síndrome, que variou de $4 \%$ o a $11 \%$, e cursou com taxa de mortalidade neonatal de $12 \%{ }^{12}$. 
A taxa de neomortalidade do grupo estudado no HGFUCS foi de $11,1 \%$.

Erkkola et al. (1994) ${ }^{13}$ observaram 43 casos de SAM numa população de 5.353 partos estudados consecutivamente, em que o mecônio no líquido amniótico esteve presente em $8,7 \%$. A duração média das gravidezes foi de $39,2 \pm 2,6$ semanas, e o peso dos RN foi de $3.570 \pm 692 \mathrm{~g}$, semelhante ao grupo controle. A taxa de cesárea no grupo com SAM foi significantemente maior que a do grupo controle $(40 \%$ vs $11,6 \%)$. Sessenta e nove por cento dos RN com SAM mostraram sinais de depressão durante ou após o trabalho de parto, ao passo que, no grupo controle, esses mesmos sinais ocorreram em 2,7\%. A mortalidade neonatal entre os que apresentavam SAM foi zero. Em seguimento de longo prazo (1 a 6 anos), foram observados 3 casos de seqüelas neurológicas $(7,0 \%)$. Em nossa casuística, a incidência de seqüela neurológica diagnosticada por ocasião da alta hospitalar foi de $11,1 \%$ ( 2 casos), se considerados apenas os 18 casos estudados. Entretanto, se fossem incluídos os 8 casos com parto fora do HG-FUCS, a taxa seria de $7,7 \%$, semelhante ao dos autores supracitados. A septicemia $(15,4 \%)$ e as crises convulsivas $(15,4 \%)$ também foram eventos identificados dentre as principais complicações observadas na UTIN do HG-FUCS.

Locus et al. ${ }^{14}$ relatam incidência de SAM de $4 \%$, Wiswell et al. ${ }^{15}$ encontraram incidência de $5,4 \%$ da sindrome, ao passo que Al Takroni et al. ${ }^{16}$ relataram incidência de 1 em $325(0,27 \%)$ com taxa de mortalidade neonatal de $7 \%$, Coltart et al. ${ }^{17}$ relatam que a incidência da SAM em 6 anos (38.395 nascimentos) foi de 1 em 533 nascimentos $(0,2 \%)$ e a taxa de mortalidade foi de 1 em 12 (8,3\%). Comparativamente, a incidência de SAM no HG-FUCS foi de $0,45 \%$ e a mortalidade neonatal, de $7,7 \%$.

A SAM associou-se a pequenos para a idade gestacional em estudo realizado por Yong e $\mathrm{Ho}^{5}$. A taxa de pequenos para a idade gestacional), em casos de SAM no HG-FUCS, foi de 5,6\% (1 caso).

Adhikari et al. ${ }^{9}$ associaram a primiparidade, bem como a idade superior a 35 anos, à alta incidência de mortalidade perinatal e ao grau de asfixia ao nascimento. Urbaniak et al. (1996) ${ }^{8}$ encontraram associação significante entre a SAM e a primiparidade. Usta et al. $(1995)^{10}$ relataram que o tabagismo esteve relacionado com riscos mais baixos de SAM. Em nosso estudo, as primigestas estiveram presentes em $55,6 \%$ dos casos (10 casos), ao passo que as multigestas estiveram presentes em 33,3 casos (6 casos). Por outro lado, as não fumantes foram a maioria $(77,8 \%)$.

A SAM tem sido descrita pelos diversos autores enfatizando o grau de gravidade da doença. No presente estudo observou-se estreita relação entre a sindrome e os resultados neonatais, especialmente no que diz respeito à depressão neonatal grave, dada pelo índice de Apgar, aumento da morbidade e mortalidade neonatal e incremento nas taxas de internação em UTIN. O sofrimento fetal intraparto ocorreu na grande maioria dos casos. A intensidade de mecônio no líquido âmnico parece ser fator importante na SAM, pois, quando espesso, associa-se mais fortemente à doença. Observou-se ainda relação entre índices de operação cesariana, superiores à da população geral do HG-FUCS, fetos de termo em sua maioria, bem como adequados para a idade gestacional.

\section{ABSTRACT}

Purpose: to analyze obstetrical and perinatal data in 26 cases of meconium aspiration syndrome (MAS).

Methods: a retrospective review was performed in 26 newborn records diagnosed with meconium aspiration syndrome. Patients were studied emphasizing average days in neonatal intensive care unit and main maternal findings and neonatal complications, correlating them with each other.

Results: Eighteen babies were delivered at GH-CSUF and eight out of this hospital. At this time 3,976 deliveries occurred at GH-CSUF, with an incidence of MAS of $0.45 \%$. Nine of 18 babies were born by vaginal delivery; weight was $\geq 2,500 \mathrm{~g}$ in 16 cases. One-minute Apgar score was $\geq 7$ in three cases (16.7\%), between 4 and 6 in seven cases (38.9\%), and between 0 and 3 , in eight cases (44.4\%). At 5 minutes, seven babies remained $<7$. Anoxia was the main neonatal complication (36\%). The mortality rate was $7.7 \%$ and the average hospital stay was 19.9 days.

Conclusion: MAS is a very important neonatal pathology correlated with high neonatal mortality rates, thick meconium in at least half of the cases, and with a majority of depressed newborns at delivery.

KEYWORDS: Meconium aspiration syndrome. Neonatal complications. Neonatal hypoxia.

\section{Referências}

1. Ramin KD, Leveno KJ, Kelly MA, Carmody TJ. Amniotic fluid meconium: a fetal environmental hazard. Obstet Gynecol 1996; 87:181-4.

2. Houlihan CM, Knuppel RA. Meconium-stained amniotic fluid. Current controversies. J Reprod Med 1994; 39:888-98.

3. Morais EN. Mecônio no líquido amniótico. In: Morais EN, Mauad Filho FO, editores. Medicina Materna e Perinatal. $1^{\mathrm{a}}$ ed. Rio de Janeiro: Revinter; 2000. p.207-16. 
4. National Institute of Child Health and Human Development Research Planning Workshop. Electronic fetal heart rate monitoring: research guidelines for interpretation. Am J Obstet Gynecol 1997; 177:1385-90.

5. Yong YP, Ho LY. A 3-year review of meconium aspiration syndrome. Singapore Med J 1997; 38:205-8.

6. Nathan L, Leveno KJ, Carmody TJ $3^{\text {rd }}$, Kelly MA, Sherman ML. Meconium: a 1990s perspective on an old obstetric hazard. Obstet Gynecol 1994; 83:329-32.

7. Benny PS, Malani S, Hoby MA, Hutton JD. Meconium aspiration - role of obstetric factors and suction. Aust N Z J Obstet Gynaecol 1987; 27:36-9.

8. Urbaniak KJ, McCowan LM, Townend KM. Risk factors for meconium-aspiration syndrome. Aust N Z J Obstet Gynaecol 1996; 36:401-6.

9. Adhikari M, Gouws E, Velaphi SC, Gwamanda P. Meconium aspiration syndrome: importance of the monitoring of labor. J Perinatol 1998; 18:55-60.

10.Usta IM, Mercer BM, Sibai BM. Risk factors for meconium aspiration syndrome. Obstet Gynecol 1995; 86:230-4.
11.Chishty AL, Alvi Y, Iftikhar M, Bhutta TI. Meconium aspiration in neonates: combined obstetric and paediatric intervention improves outcome. J Pak Med Assoc 1996; 46:104-8.

12.Adhikari M, Gouws E. Meconium aspiration in South Africa. S Afr Med J 1995; 85:891-3.

13.Erkkola R, Kero P, Suhonen-Polvi H, Korvenranta H. Meconium aspiration syndrome. Ann Chir Gynaecol Suppl 1994; 208:106-9.

14.Locus P, Yeomans E, Crosby U. Efficacy of bulb versus DeLee suction at deliveries complicated by meconium stained amniotic fluid. Am J Perinatol 1990; 7:87-91.

15.Wiswell TE, Tuggle JM, Turner BS. Meconium aspiration syndrome: have we made a difference? Pediatrics 1990; 85:715-21.

16.Al Takroni AM, Parvathi CK, Mendis KB, Hassan S, Reddy I, Kudair HA. Selective tracheal suctioning to prevent meconium aspiration syndrome. Int $\mathrm{J}$ Gynaecol Obstet 1998; 63:259-63.

17. Coltart TM, Byrne DL, Bates SA. Meconium aspiration syndrome: a 6-year retrospective study. Br J Obstet Gynaecol 1989; 96:411-4.

Recebido em: 17/10/2001 Aceito após modificações em: 22/3/2003

\section{AVISO:}

\section{Solicitamos aos associados manter o}

\section{endereço para correspondência atualizado}

junto a sua Federada e a FEBRASGO a fim de receber regularmente nossas publicações. 